\title{
NF-YA promotes invasion and angiogenesis by upregulating EZH2-STAT3 signaling in human melanoma cells
}

\author{
ZIHAN XU, YAOWEN SUN, YADONG GUO, GAOPING QIN, SHENGZHI MU, RONGHUI FAN, \\ BENFENG WANG, WENJIE GAO, HANGLI WU, GUODONG WANG and ZHENXIN ZHANG \\ Department of Burns and Plastic Surgery, Provincial People's Hospital, \\ Xi'an, Shaanxi 710068, P.R. China
}

Received December 11, 2015; Accepted February 22, 2016

DOI: $10.3892 /$ or.2016.4761

\begin{abstract}
The process of angiogenesis is essential for tumor development and metastasis. Vascular endothelial growth factor (VEGF), which is overexpressed in most human cancers, has been demonstrated to be a major modulator of angiogenesis. Thus, inhibition of VEGF signaling has the potential for tumor anti-angiogenic therapy. Signal transducer and activator of transcription-3 (STAT3) is a key regulator for angiogenesis by directly binding to the VEGF promoter to upregulate its transcription. Several factors can enhance STAT3 activity to affect angiogenesis. Here, we found that overexpression of nuclear transcription factor-Y alpha (NF-YA) gene could promote cell invasion and angiogenesis accompanying the increase of STAT3 signaling in human melanoma cells. Moreover, the expression and secretion of VEGF was also found to be upregulated by the overexpression of NF-YA gene in melanoma cells. The STAT3 inhibitor was able to attenuate the upregulation of VEGF induced by NF-YA overexpression. Enhancer of zeste homolog 2 (EZH2), the catalytic subunit of the Polycomb repressive complex 2 , enhances STAT3 activity by mediating its lysine methylation. We also showed that NF-YA upregulated the expression of EZH2 and NF-YA-induced angiogenesis could be inhibited by EZH2 knockdown. Taken together, these findings indicate that overexpression of NF-YA contributes to tumor angiogenesis through EZH2-STAT3 signaling in human melanoma cells, highlighting NF-YA as a potential therapeutic target in human melanoma.
\end{abstract}

Correspondence to: Professor Zhenxin Zhang, Department of Burns and Plastic Surgery, Provincial People's Hospital, 256 West Youyi Road, Xi'an, Shaanxi 710068, P.R. China

E-mail: zzsxa2006@163.com

Key words: nuclear transcription factor-Y alpha, angiogenesis, vascular endothelial growth factor, STAT3, enhancer of zeste homolog 2

\section{Introduction}

Melanoma is an aggressive and highly metastatic tumor with poor prognosis and an increasing incidence rate in recent decades $(1,2)$. The process of angiogenesis is a key step essential for tumor development and metastasis, including melanoma (3). Vascular endothelial growth factor (VEGF), a major angiogenic factor for vascular endothelial cells, is overexpressed in most human cancers $(4,5)$. Targeting VEGF signaling pathway is a rapidly growing chemotherapy class for tumor anti-angiogenic therapy $(6,7)$. Thus, a better understanding of the regulation of VEGF by other factors would provide more effective strategies for the development of new anti-angiogenic therapy.

Tumor hypoxia is a common feature of the microenvironment of many solid tumors $(8,9)$. VEGF expression is well known to be upregulated by hypoxia $(10,11)$. Hypoxia-inducible factor 1 (HIF-1) is responsible for the transcriptional activation of the VEGF gene in cells exposed to hypoxia by binding to a hypoxia response element of VEGF gene promoter $(12,13)$. Signal transducer and activator of transcription-3 (STAT3) was originally described as latent cytoplasmic transcription factor in response to extracellular signaling proteins $(14,15)$. STAT3 is activated by phosphorylation in response to various cytokines and growth factors and is constitutively activated with high frequency in diverse human cancer cell lines and tissues (16). STAT3 has also been shown to be a transcriptional activator of the VEGF gene by directly binding to the VEGF promoter to upregulate its transcription (17). Enhancer of zeste homolog 2 (EZH2), a catalytic subunit of the Polycomb repressive complex 2, catalyzes the trimethylation of lysine 27 in histone 3 (H3K27me3) and facilitates heterochromatin formation thereby silences gene transcription $(18,19)$. Interestingly, EZH2 can also methylate non-histone proteins such as the transcription factor nuclear receptor RORA, Talin-1 and STAT4 (20-22). Moreover, EZH2 can directly bind to STAT3 protein and then mediate its lysine methylation to enhance STAT3 activity (23).

Nuclear transcription factor-Y alpha (NF-YA) is a transcription factor that specifically binds to CCAAT motifs of the promoter regions in a variety of genes (24). By the binding to CCAAT motifs in the promoter of genes, NF-YA can serve as either an activator or a repressor in the transcriptional regula- 
tion of its target genes (25). NF-YA target genes were found to be involved in cell proliferation (26), DNA repair and cell death $(27,28)$. However, the role of NF-YA in invasion and angiogenesis of tumor cells has not been reported.

Here we demonstrate that NF-YA is upregulated in human melanoma cell lines with highly invasive potential. Overexpression of NF-YA promotes cell invasion and angiogenesis of human melanoma cells. Moreover, the expression and secretion of VEGF can be upregulated by the overexpression of NF-YA gene in melanoma cells. The activity of STAT3 is responsible for VEGF upregulation (17). We also show that NF-YA can induce the activity of STAT3 and NF-YA-induced upregulation of VEGF can be inhibited by STAT3 inhibitor. Furthermore, knock-down of NF-YA target gene EZH2 also attenuates the angiogenesis induced by the overexpression of NF-YA. Our results suggest that NF-YA contributes to tumor angiogenesis through EZH2-STAT3 signaling in human melanoma cells, and that NF-YA is therefore a potential therapeutic target for treatment of human melanoma.

\section{Materials and methods}

Reagents. STAT3 inhibitor WP1066 (working concentration: $5 \mu \mathrm{M}$ ) was purchased from Selleck Chemicals (cat no. S2796; Houston, TX, USA). The mouse anti-human monoclonal antibodies to NF-YA (cat no. sc-17753; 1:1,000 dilution), STAT3 (cat no. sc-293151; 1:1,000 dilution), EZH2 (cat no. sc-166609; $1: 1,000$ dilution) and $\beta$-actin (cat no. sc-8432; 1:2,000 dilution) were from Santa Cruz Biotechnology, Inc. (Dallas, TX, USA). Mouse anti-human monoclonal antibody to tyrosine phosphorylated STAT3 (pY-STAT3, at the 705 residue; cat no. \#9138; 1:1,000 dilution) was from Cell Signaling Technology, Inc. (Danvers, MA, USA). Mouse anti-human monoclonal antibody to VEGF (cat no. ab155932; 1:1,000 dilution) was from Abcam (Cambridge, MA, USA). Rabbit anti-methylated lysine polyclonal antibody (Methy-K, cat no. ADI-KAP-TF121-E; 1:1,000 dilution) was from Enzo Life Sciences (Farmingdale, NY, USA). Horseradish peroxidase (HRP)-labeled goat anti-mouse IgG antibody (cat no. ab6789) and goat anti-rabbit IgG antibody (cat no. ab6721) were from Abcam. Protein A/G PLUS-Agarose was from Santa Cruz Biotechnology, Inc. (cat no. sc-2003). Alexa-568-labeled goat anti-mouse secondary antibodies (cat no. A-11031; Thermo Fisher Scientific Inc. Kalamazoo, MI, USA). 4,6-diamidino-2-phenylindole (DAPI) was from Sigma (cat no. D9542; St. Louis, MO, USA).

Cell culture. A375P, Malme-3M, A375-M2 and SK-MEL-28 human melanoma cell lines were purchased from the American Type Culture Collection (ATCC; Manassas, VA, USA). Human umbilical vein endothelial cells (HUVECs) and HEK293T cell lines were from the Institute of Cell and Biochemistry Research of the Chinese Academy of Science (Shanghai, China). HEK293T, A375P, Malme-3M, A375-M2 and SK-MEL-28 cells were cultured with Dulbecco's modified Eagle's medium (DMEM; Hyclone, Logan, UT, USA) supplemented with 10\% fetal bovine serum (Gibco, Invitrogen, Grand Island, NY, USA) in a humidified atmosphere containing $5 \% \mathrm{CO}_{2}$ at $37^{\circ} \mathrm{C}$. HUVECs were cultured in Kaighn's modified Ham's F-12K medium (Thermo Fisher Scientific, Inc., Waltham, MA, USA) containing 10\% fetal bovine serum (Gibco, Invitrogen), $0.1 \mathrm{mg} / \mathrm{ml}$ heparin (American Pharmaceutical Partners, Schaumburg, IL, USA), and 0.03-0.05 mg/ml ECGS (BD Biosciences, Franklin Lakes, $\mathrm{NJ}, \mathrm{USA}$ ), at $37^{\circ} \mathrm{C}$ and $5 \%$ humidified atmospheric $\mathrm{CO}_{2}$.

Plasmids. The human NF-YA (NM_002505) gene stably overexpressing vector using lentiviral vector pCDHCMV-MCS-EF1-Puro (System Biosciences, Mountain View, CA, USA) was constructed by Genesent Biotech (Shanghai, China). The shRNA lentiviral vector pLKO.1 was from Open Biosystems (Huntsville, AL, USA). Lentivirus vectors pLKO. 1 containing short hairpins against NF-YA or EZH2 or scrambled shRNA controls were purchased from Sigma. The target sequences of the two genes were used as follows: NF-YA, AGTCCAAGGGCAGCCATTAAT (sh-1-N) and CCATCA TGCAAGTACCTGTTT (sh-2-N); EZH2, CCCAACATAGAT GGACCAAAT (sh-1-E) and CCCAACATAGATGGACCA AAT (sh-2-E).

Lentivirus production and transduction. For lentiviral production, HEK293T cells $\left(5 \times 10^{6}\right)$ were co-transfected with lentiviral plasmids, packaging vector pCMV-dR8.2 dvpr, and VSV-G (Addgene, Cambridge, MA, USA) to produce lentiviral particles. The lentiviral particles were harvested after $48 \mathrm{~h}$ by centrifugation and concentrated using the Lenti ${ }^{\mathrm{TM}}$ concentrator kit (Clontech, Lonza, Wokingham, UK). The lentiviral particles were added to target cells and incubated for $24 \mathrm{~h}$ in the presence of $8 \mu \mathrm{g} / \mathrm{ml}$ of polybrene. Lentivirus-transduced cells were then selected with puromycin for 4 days to generate cells with stable overexpression or knockdown of the target genes. The expression of the target gene was detected by western blot analysis.

$R N A$ purification and $R T-q P C R$. Total RNA was isolated from cultured cells with TRIzol reagent (Invitrogen, Carlsbad, CA, USA) for RNA extraction. The reverse transcription was carried out with a PrimeScript Reverse Transcriptase reagent kit (Takara, Japan). Real-time quantitative PCR analysis was performed using SYBR Green Master Mix (Takara) according to the manufacturer's instructions. The mRNA of $\beta$-actin was treated as normalization control. Primers were used as follows: NF-YA, 5'-GGCAGACCATCGTCTATCAACC-3' sense and 5'-ATCTGTGCTCCTGCCAAACTGG-3', antisense; VEGF, 5'-TTGCCTTGCTGCTCTACCTCCA-3' sense and 5'-GATG GCAGTAGCTGCGCTGATA-3', antisense; EZH2, 5'-GACCT CTGTCTTACTTGTGGAGC-3' sense and 5'-CGTCAGATG GTGCCAGCAATAG-3', antisense; and $\beta$-actin, 5'-CACCATT GGCAATGAGCGGTTC-3' sense and 5'-AGGTCTTTGCGG ATGTCCACGT-3', antisense. All real-time amplifications were performed in the ABI 7900 Fast Real-Time PCR system (Applied Biosystems, Foster City, CA, USA). Results of the real-time PCR data were represented as $\mathrm{Ct}$ values of target genes normalized to its averaged $\mathrm{Ct}$ values of $\beta$-actin gene to give a $\Delta \mathrm{Ct}$ value.

Western blot analysis and immunoprecipitation. For western blot analysis, cells were washed twice with PBS and then lysed using $200 \mu \mathrm{l}$ of RIPA lysis buffer for $30 \mathrm{~min}$ on ice. The quantity of protein was measured using a BCA protein assay (Bio-Rad, Hercules, CA, USA). Samples were then separated 
on SDS-PAGE and transferred to nitrocellulose membranes. The membranes were blocked with 5\% BSA in TBST for $1 \mathrm{~h}$ at room temperature. The membranes were then incubated with specific primary antibodies overnight at $4^{\circ} \mathrm{C}$. The antibodies to NF-YA, STAT3, pY-STAT3, VEGF, Methy-K, and EZH2 were used at a 1:1,000 dilution. The antibody to $\beta$-actin were used at a 1:2,000 dilution. Horseradish peroxidase (HRP)-labeled goat anti-mouse IgG antibody and goat anti-rabbit IgG antibody were used at a 1:5,000 dilution. The specifically bound antibodies were detected by chemiluminescence (ECL) reagent (ECL-Plus kit; Amersham, Piscataway, NJ, USA) and visualized with Fujifilm LAS-3000 (Multi Gauge; Fujifilm, Tokyo, Japan). For immunoprecipitation to purify STAT3 protein, cells were lysed in lysis buffer $(50 \mathrm{mM}$ Tris- $\mathrm{HCl}$ at $\mathrm{pH} 8.0$, $150 \mathrm{mM} \mathrm{NaCl}, 2 \mathrm{mM}$ EDTA and $0.1 \%$ NP-40) supplemented with protease and phosphatase inhibitors (cat no. 1873580 and 4906837001; Roche Diagnostics, Basel, Switzerland) at $4^{\circ} \mathrm{C}$ for $30 \mathrm{~min}$ and cleared by centrifugation at $14,000 \mathrm{rpm}$ at $4^{\circ} \mathrm{C}$. The cleared lysate was incubated for $6 \mathrm{~h}$ at $4^{\circ} \mathrm{C}$ with agarose-conjugated STAT3 antibody.

ELISA analysis. The supernatants of the cells were assayed for VEGF using appropriate sandwich ELISA kit (cat no. SVE00; R\&D Systems, Minneapolis, MN, USA). The cells were incubated in 24-well plates (Corning Costar, Corning, NY, USA) for $12 \mathrm{~h}$. The medium was replaced with serum-free medium for $24 \mathrm{~h}$. VEGF concentrations were determined by measuring absorbance at $420 \mathrm{~nm}$. VEGF values were normalized to the total protein extracted from wells.

Matrigel invasion assay. Cells $\left(5 \times 10^{5}\right)$ were cultured in serum-free DMEM for $12 \mathrm{~h}$ and then seeded into the upper chambers (24-well, pore size $8-\mu \mathrm{m}$; Millipore, Billerica, MA, USA) coated with a layer of Matrigel (BD Biosciences, San Jose, CA, USA). Following 24-h incubation at $37^{\circ} \mathrm{C}$, the cells that did not invade through the pores were removed from the top chamber by scraping with cotton swab. The invading cells were fixed with methanol and stained with hematoxylin-eosin (H\&E) and counted. The number of invasive cells were counted under a microscope (magnification, x100).

Immunofluorescence staining. The vector control or NF-YA over-expressed A375P melanoma cells were seeded onto glass slides and incubated at $37^{\circ} \mathrm{C}$ with $5 \% \mathrm{CO}_{2}$. Attached for $24 \mathrm{~h}$, cells were then fixed with $37 \%$ formaldehyde in PBS, permeabilized for $10 \mathrm{~min}$ in PBS containing $0.5 \%$ Triton $\mathrm{X}-100$ and $1 \%$ bovine serum albumin (BSA). Then, fixed cells were incubated for $1 \mathrm{~h}$ in blocking buffer (5\% BSA with $0.1 \%$ Triton X-100 in PBS). The slides were then incubated with anti-pY-STAT3 (1:500) at $4^{\circ} \mathrm{C}$ overnight followed by incubation with Alexa-568-labeled secondary antibodies. Nuclear Staining was used with DAPI. Images were obtained using fluorescence microscopy.

Tube formation assay. To perform the tube formation assay, $5 \times 10^{4}$ HUVECs were plated in the upper chamber coated with Matrigel (BD Biosciences) followed by incubation at $37^{\circ} \mathrm{C}$ overnight. The human melanoma cells were grown in the lower chamber. At confluence for melanoma cells, the medium was changed to serum-free medium. Cultured for 3 days, the tube-like structures that formed in the gel were photographed (magnification, x100).

Statistical analysis. The data were analyzed using GraphPad Prism 5.0 (GraphPad Software, La Jolla, CA, USA). Statistical analysis was performed using a two-tailed Student's t-test. Results of $\mathrm{P}<0.05$ were considered to be significant.

\section{Results}

NF-YA is upregulated in highly invasive human melanoma cells and promotes cell invasion. To examine the role of NF-YA in human melanoma cells, we first screened four human melanoma cell lines with different invasive capacities. Western blot analysis indicated that the expression of NF-YA was detectable in all of these cell lines (Fig. 1A). By performing real-time PCR, we also found that the NF-YA mRNA expression patterns were consistent with the protein expression pattern (Fig. 1A). Malme-3M, A375P, SK-MEL-28 and A375-M2 human melanoma cell lines differ in their invasive properties. A375P cell lines are poorly metastatic, Malme-3M cell lines are moderately metastatic, while A375-M2 and SK-MEL-28 are highly metastatic (29-31). The data show that NF-YA expression patterns are consistent with the invasive potentials of these human melanoma cell lines. Next, we stably over-expressed NF-YA gene in A375P and Malme-3M cells (Fig. 1B). As shown in Fig. 1B, melanoma cells with NF-YA overexpression exhibited significant increase of cell invasion, whereas, knock-down of NF-YA significant inhibited the invasion of A-375-M2 and SK-MEL-28 cells (Fig. 1B).

NF-YA promotes angiogenesis induced by melanoma cells. Invasive tumor cells usually increase vascular endothelial cell proliferation and angiogenic activity. As a transcriptional factor, NF-YA may be involved in the regulation of angiogenesis to vascular endothelial cells by NF-YA target genes. Thus, we performed a tube formation assay in human umbilical vein endothelial cells (HUVECs) co-cultured with melanoma cells. HUVECs were co-cultured with vector control or NF-YA stably over-expressed A375P or Malme-3M melanoma cells through the collagen matrix. As shown in Fig. 2A, HUVECs co-cultured with NF-YA stably overexpressed melanoma cells exhibited significantly increased tubular networks formation compared with the vector control. Additionally, HUVECs co-cultured with NF-YA stably knock-down melanoma cells exhibited significantly decreased tubular networks formation compared with the scramble control (Fig. 2A). Vascular endothelial growth factor (VEGF) is a major modulator to angiogenesis (6). Here, we also found that the secretion of VEGF was augmented by NF-YA stably overexpressed melanoma cells (Fig. 2B).

NF-YA upregulates the expression of VEGF and increases the activity of STAT3. Elevated VEGF secretion was found in NF-YA stably overexpressed melanoma cells together with an increased tubular networks formation. We then performed western blot analysis and real-time PCR to examine whether NF-YA affect the expression of VEGF in protein and transcription levels. Overexpression of NF-YA significantly increased the expression of VEGF in A375P 
A
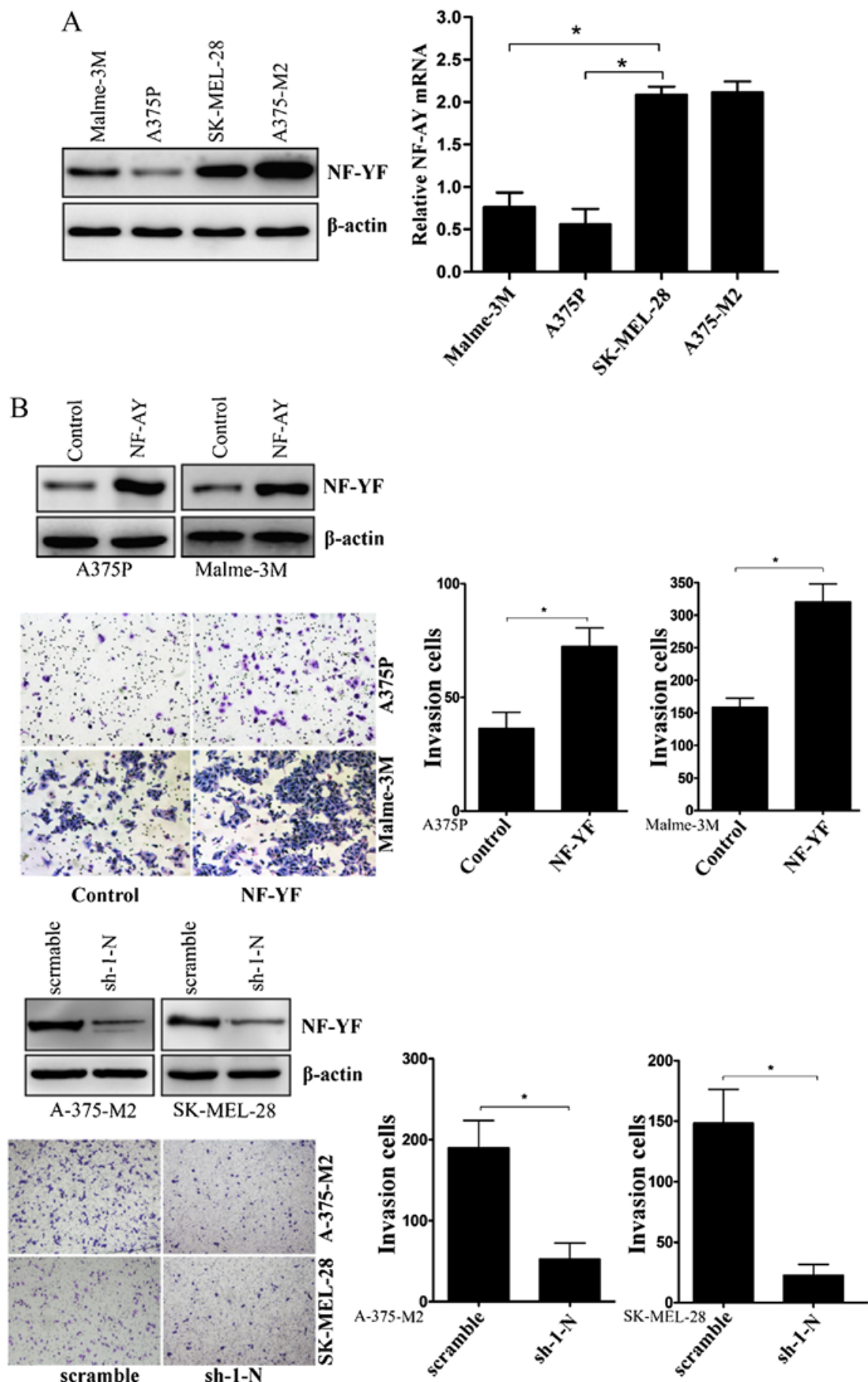

Figure 1. NF-YA is upregulated in highly invasive human melanoma cells and promotes cell invasion. (A) Left: NF-YA protein levels in human melanoma cell lines by western blot analysis; right: relative mRNA expression of NF-YA by real-time quantitative PCR in human melanoma cell lines, ${ }^{\mathrm{P}}<0.003$ compared with the control group (SK-MEL-28). (B) NF-YA protein levels in vector control and NF-YA stably overexpressed A375P/Malme-3M cells or in scramble control and NF-YA stably knock-down A-375-M2/SK-MEL-28 cells by western blot analysis. These cells were seeded in a Matrigel-coated upper chamber. Cells invaded through the Matrigel after incubation for $24 \mathrm{~h}$ was detected by $\mathrm{H} \& \mathrm{E}$ staining and quantified (lower panel). Data expression was analyzed by quantification of six randomly selected fields. " $\mathrm{P}<0.001$ compared with the control group. H\&E, hematoxylin and eosin.

or Malme-3M melanoma cells at both the transcription and protein levels (Fig. 3A). To further investigate the signaling pathway that mediated VEGF overexpression, we detected the activity of STAT3 in NF-YA overexpressed cells. STAT3 is a latent transcription factor, which is phosphorylated in active forms. Active STAT3 directly regulates the promoter of the VEGF gene and up-regulates its expression (17). Tyrosine phosphorylated STAT3 at the 705 residue (pY-STAT3) is a constitutively active form of STAT3 (32). We assessed the expression of total and phosphorylated STAT3 (pY-STAT3) in vector control and NF-YA overexpressed melanoma cells with western blot. We found that the STAT3 total expression levels remained unchanged after overexpression of NF-YA in melanoma cells, whereas STAT3 phosphorylation was enhanced (Fig. 3B). Moreover, immunofluorescence analysis on A375P melanoma cells indicated increased number of 


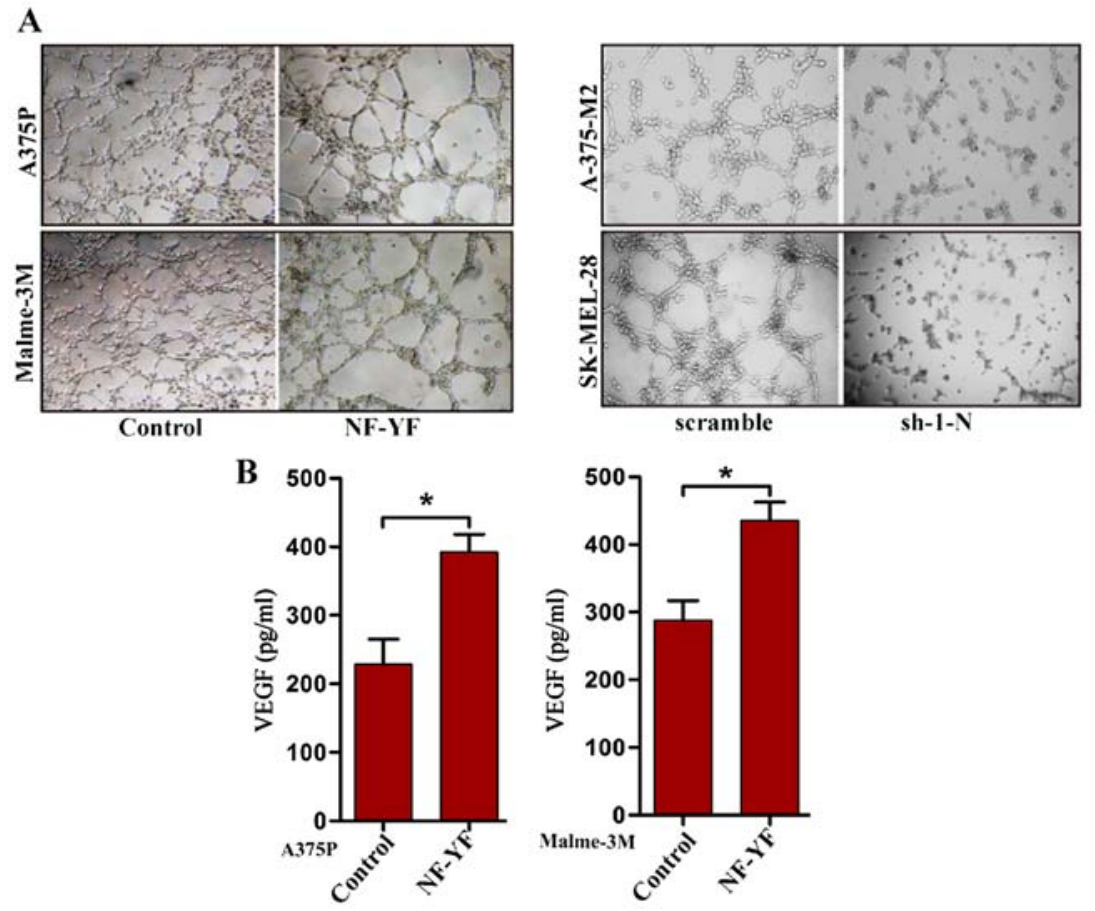

Figure 2. NF-YA promotes angiogenesis. (A) HUVECs were plated in the upper chamber coated with Matrigel in Transwell filters ( $0.4 \mu \mathrm{m})$. The vector control and NF-YA stably overexpressed A375P/Malme-3M cells or scramble control and NF-YA stably knock-down A-375-M2/SK-MEL-28 cells were grown in the lower chamber. Cultured for 3 days, the tube-like structures that formed in the gel were photographed (magnification, $x 100$ ). (B) Secretion of VEGF in the culture medium from vector control or NF-YA stably overexpressed A375P/Malme-3M cells was determined by ELISA. "P<0.005 compared with the control group.

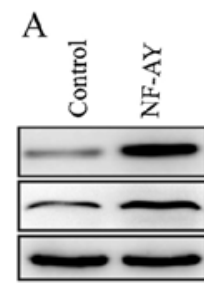

A375P

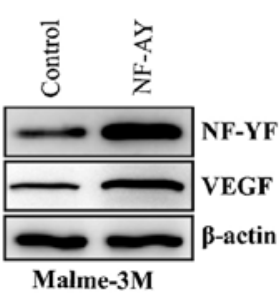

Malme-3M

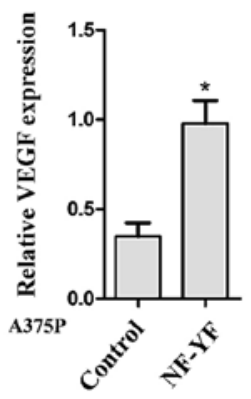

pY-STAT3

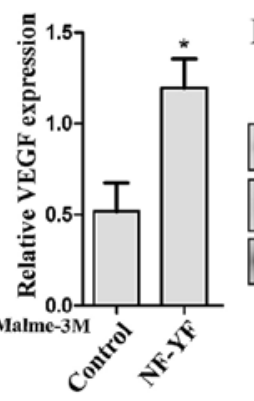

B

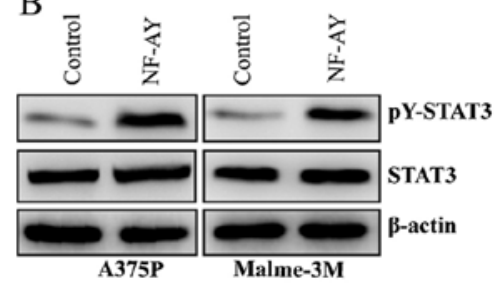

C

DAPI

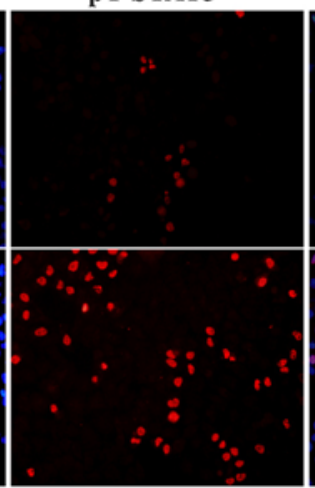

merge

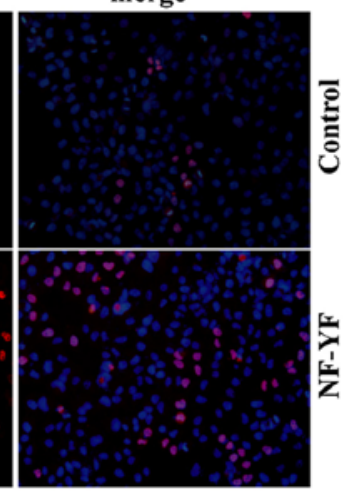

Figure 3. NF-YA increases the expression of VEGF and activity of STAT3. (A) NF-YA, VEGF and $\beta$-actin protein levels were detected by western blot analysis in vector control or NF-YA stably overexpressed A375P/Malme-3M cells (left panel); relative mRNA expression of VEGF in vector control or NF-YA stably overexpressed A375P/Malme-3M cells by real-time quantitative PCR (left panel), ${ }^{*} \mathrm{P}<0.003$ compared with the control group. (B) STAT3, pY-STAT3 (tyrosine phosphorylated STAT3 at the 705 residue) and $\beta$-actin protein levels were detected by western blot analysis in vector control or NF-YA stably overexpressed A375P/Malme-3M cells. (C) Immunofluorescence staining showing expression of pY-STAT3 (red) in vector control or NF-YA stably overexpressed A375P cells. Nuclei were stained with DAPI (blue).

pY-STAT3 positive cells in NF-YA overexpressed cells compared to the vector control (Fig. 3C).
NF-YA induced upregulation of VEGF can be attenuated by STAT3 inhibition. Because our data indicate that 

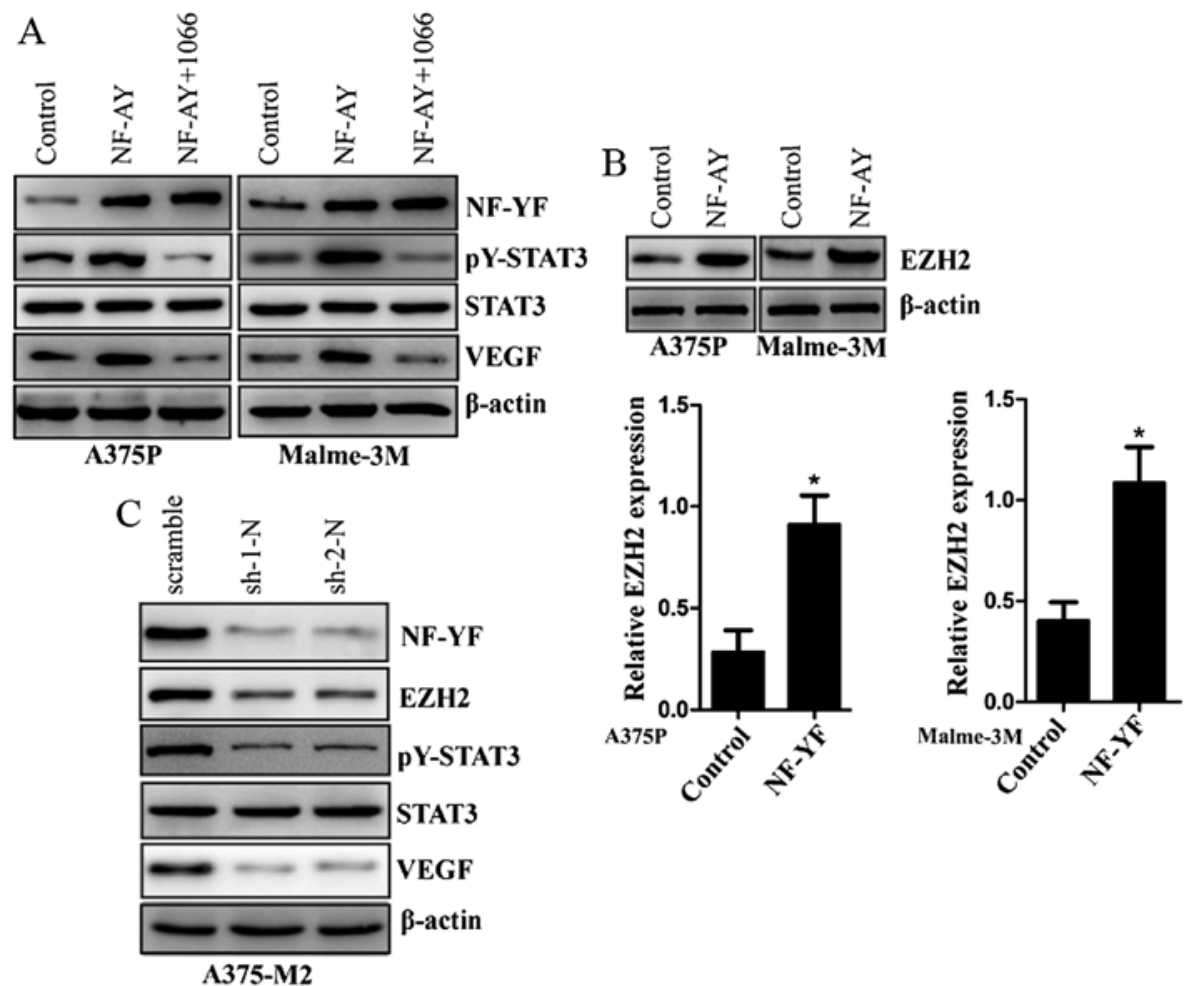

Figure 4. NF-YA upregulated VEGF in a STAT3-dependent manner. (A) NF-YA, STAT3, pY-STAT3, VEGF and $\beta$-actin protein levels were detected by western blot analysis in vector control or NF-YA stably overexpressed (or NF-YA stably overexpressed plus STAT3 inhibitor WP1066 treatment) A375P/Malme-3M cells. (B) EZH2 and $\beta$-actin protein levels were detected by western blot analysis in vector control or NF-YA stably overexpressed A375P/Malme-3M cells (upper panel). Relative mRNA expression of VEGF in vector control or NF-YA stably overexpressed A375P/Malme-3M cells by real-time quantitative PCR (low panel), "P<0.004 compared with the control group. (C) NF-YA, EZH2, STAT3, pY-STAT3, VEGF and $\beta$-actin protein levels were detected by western blot analysis in scramble control or NF-YA stably knockdown (sh-1-N, sh-2-N) A375-M2 cells.

overexpression of NF-YA upregulates the expression of VEGF and increases the activity of STAT3, we investigated a possible downstream factor of NF-YA to mediate this effect. WP1066 is a STAT3 inhibitor by blocking constitutively activated STAT3 phosphorylation (Tyr 705 residue) $(32,33)$. We further examined the role of WP1066 in NF-YA stably overexpressed melanoma cells. With the treatment of WP1066 in NF-YA stably overexpressed A375P or Malme-3M melanoma cells, NF-YA induced upregulation of VEGF and increased activity of STAT3 was inhibited (Fig. 4A). EZH2 was reported to directly bind to STAT3 protein and then mediate its lysine methylation to enhance STAT3 activity (23). Actually, NF-YA is a key regulator of EZH2 expression (34). In NF-YA stably over-expressed A375P and Malme-3M melanoma cells, we confirm that the expression of EZH2 is upregulated by the overexpression of NF-YA at both transcription and protein levels (Fig. 4B). In addition, we sought to determine the effects of NF-YA knockdown in melanoma cells. Stable knockdown of NF-YA in A375-M2 melanoma cells resulted in downregulated expression of EZH2 and VEGF (Fig. 4C), and the activity of STAT3 was also inhibited in A375-M2 melanoma cells with NF-YA stable knockdown (Fig. 4C).

NF-YA upregulates VEGF expression through activating EZH2-STAT3 signaling. Since NF-YA knockdown suppresses the expression of EZH2 and the activity STAT3, we examined whether knockdown of NF-YA inhibits the lysine methylation of STAT3 in A375-M2 cells. STAT3 protein from control or NF-YA knockdown A375-M2 cells were immunoprecipitated with anti-STAT3 antibody and the blots were analyzed for methylated STAT3 using pan-methyl lysine antibody. The methylation of STAT3 protein from NF-YA knockdown A375-M2 cells was significantly reduced compared with the scramble control A375-M2 cells (Fig. 5A). The enhanced STAT3 activity was mediated by its lysine methylation. Consistent with the methylation of STAT3, STAT3 phosphorylation (Tyr 705 residue) was also inhibited in NF-YA knockdown A375-M2 cells (Fig. 5A). Next, we determined whether EZH2 knockdown could affect the expression of NF-YA. The result showed that EZH2 knockdown did not affect the expression of NF-YA in A375P cells (Fig. 5B). Differently, knockdown of EZH2 inhibited the expression of VEGF, the methylation and activity of STAT3 (Fig. 5B). Together, these data suggest that NF-YA regulates STAT3 activity and VEGF expression via upregulating EZH2 expression. To further confirm this, we overexpressed NF-YA in EZH2 knockdown A375P cells. Although NF-YA was overexpressed in EZH2 knockdown A375P cells, the expression of VEGF and the activity of STAT3 did not change (Fig. 5B). Likely, NF-YA did not affect the cell invasion of A375P cells with EZH2 knockdown (Fig. 5C). HUVECs co-cultured with EZH2 knockdown A375P cells did not exhibit significant tubular networks formation by the overexpression of NF-YA in these EZH2 knockdown cells (Fig. 5D). 

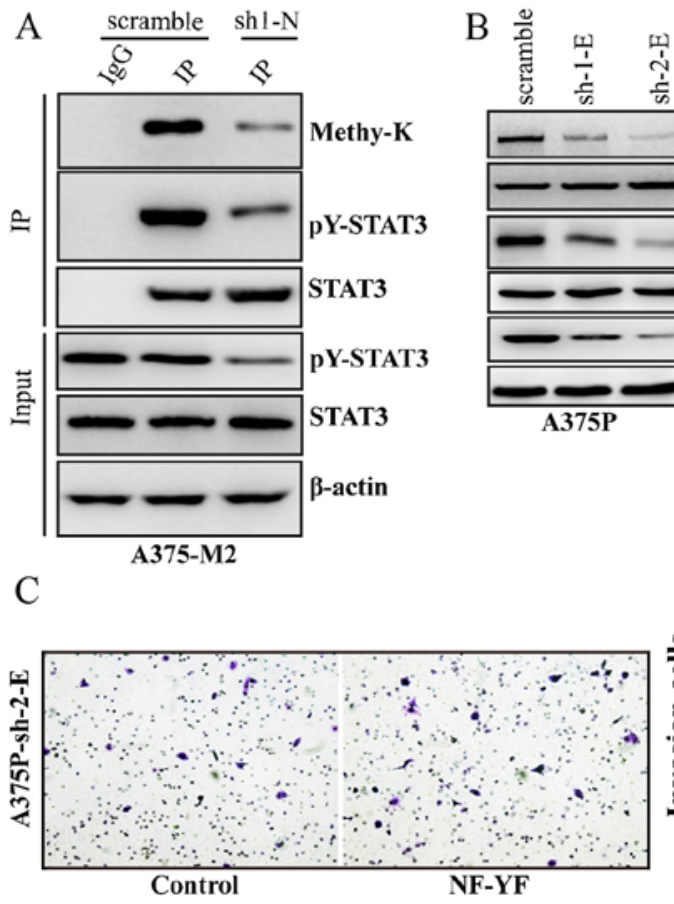
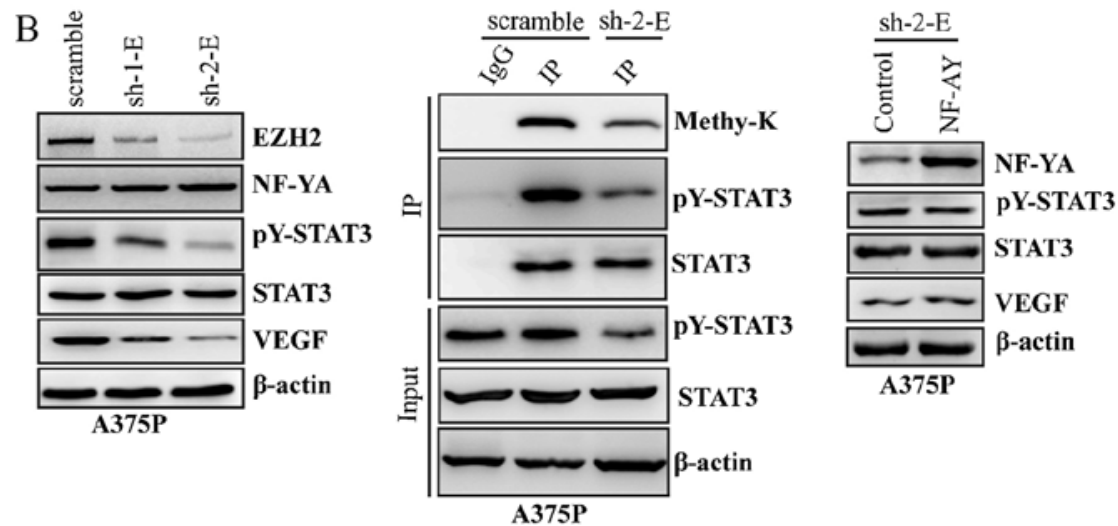

D

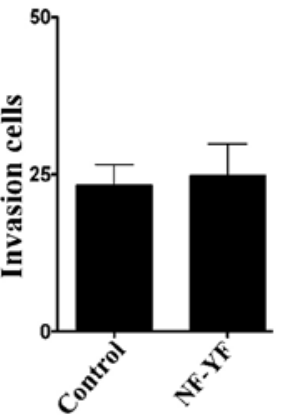

Figure 5. The role of EZH2 in NF-YA induced VEGF expression and STAT3 activation. (A) Immunoprecipitation of STAT3 protein using antibodies for STAT3 (IP), or a non-specific immunoglobulin G (IgG) from scramble control or NF-YA stably knockdown (sh-1-N) A375-M2 cells, followed by immunoblots with the indicated antibodies. Methy-K, means rabbit anti-methylated lysine antibody; input indicate 5\% of pre-immunoprecipitated samples. (B) NF-YA, EZH2, STAT3, pY-STAT3, VEGF and $\beta$-actin protein levels were detected by western blot analysis in scramble control or EZH2 stably knockdown (sh-1-E, sh-2-E) A375P cells (left panel); immunoprecipitation of STAT3 protein using antibodies for STAT3 (IP), or a non-specific immunoglobulin G (IgG) from scramble control or EZH2 stably knockdown (sh-2-E) A375P cells, followed by immunoblots with the indicated antibodies. Methy-K, means rabbit antimethylated lysine antibody; input indicate $5 \%$ of pre-immunoprecipitated samples (middle panel); NF-YA, STAT3, pY-STAT3, VEGF and $\beta$-actin protein levels were detected by western blot analysis in vector control and NF-YA stably overexpressed A375P cells with EZH2 stably knockdown (sh-2-E) (right panel). (C) The vector control or NF-YA stably overexpressed A375P-sh-2-E cells were seeded in a Matrigel-coated upper chamber. Cells invaded through the Matrigel after incubation for $24 \mathrm{~h}$ was detected by H\&E staining and quantified (lower panel). Data expression was analyzed by quantification of six randomly selected fields. A375P-sh-2-E, means A375P cells with EZH2 stably knockdown (sh-2-E). (D) HUVECs cells were plated in the upper chamber coated with Matrigel in Transwell filters $(0.4 \mu \mathrm{m})$. The vector control or NF-YA stably overexpressed A375P-sh-2-E cells were grown in the lower chamber. Cultured for 3 days, the tube-like structures that formed in the gel were photographed (magnification, $\mathrm{x} 100$ ).

\section{Discussion}

Melanoma is an aggressive and highly metastatic tumor with a high angiogenic potential (1). Angiogenesis is triggered by molecular signals from tumor cells when tumor tissues require nutrients and oxygen (35). A number of growth factors stimulate angiogenesis by inducing endothelial cell migration (4), proliferation (6) and expansion (36). These growth factors include vascular endothelial growth factor (VEGFs), basic fibroblast growth factor (bFGF), hepatocyte growth factor (HGF), platelet-derived growth factor (PDGF), transforming growth factors $\beta$ (TGF $\beta$ ), interleukin-6 (IL-6), interleukin-8 (IL-8) and epidermal growth factor (EGF) $(35,37)$. Among these, VEGF (also known as VEGF-A) is a protein with vascular permeability activity that was first identified as a factor secreted by tumor cells $(38,39)$. VEGF is a well-studied and powerful angiogenic factor. Anti-angiogenic therapy targeting VEGF signaling is a rapidly growing chemotherapy class for cancer therapy (6). Thus, a better understanding of the regulation of VEGF by other factors would provide more effective strategies for the development of new anti-angiogenic therapy.
The expression of VEGF is well known to be upregulated by hypoxia (13). Hypoxia induces the expression of VEGF via hypoxia-inducible factor- $1 \alpha$ (HIF-1 $\alpha)$ by binding to a hypoxia response element of VEGF gene promoter to activate VEGF gene expression in cells exposed to hypoxia (11). Moreover, STAT3 also has been shown to be a transcriptional activator of the VEGF gene by directly binding to the VEGF promoter (12). In the present study, we demonstrated that NF-YA increases the expression of VEGF and promotes angiogenesis of human melanoma cells in vitro. The results suggest that targeting NF-YA has the potential for tumor antiangiogenic therapy.

EZH2 was reported to directly bind to STAT3 protein and mediate its lysine methylation to enhance STAT3 activity (23). Moreover, NF-YA is a key regulator of EZH2 expression by up-regulating its transcription (34). Consistent with this report, we also showed that overexpression of NF-YA in human melanoma cells up-regulated the expression of EZH2. Because our data indicate that NF-YA upregulates the expression of VEGF and increases the activity of STAT3, STAT3 inhibitor WP1066 was used to study the role of STAT3 in NF-YA induced up-regulation of VEGF. With the treatment of WP1066 in 
NF-YA stably overexpressed A375P or Malme-3M melanoma cells, NF-YA induced upregulation of VEGF and the increased activity of STAT3 was inhibited.

EZH2-induced lysine methylation of STAT3 is a critical modification that leads to STAT3 activation (23). In the present study, we observed that the lysine methylation of STAT3 protein from NF-YA knockdown A375-M2 cells was significantly reduced compared with the control A375-M2 cells. Consistent with the methylation of STAT3, STAT3 phosphorylation (Tyr 705 residue) was also inhibited in NF-YA knockdown A375-M2 cells. Furthermore, knockdown of EZH2 was shown to inhibit the expression of VEGF and phosphorylation of STAT3 in A375P cells. Importantly, the expression of VEGF and phosphorylation of STAT3 were not affected by the overexpression of NF-YA in EZH2 stably knockdown A375P cells. Similarly, NF-YA did not affect the cell invasion and angiogenesis of A375P cells with stable EZH2 knockdown.

Taken together, the present study demonstrated that overexpression of NF-YA contributes to cell invasion and angiogenesis through EZH2-STAT3 signaling in human melanoma cells. Therefore, our results suggest the possibility that NF-YA might be a potential therapeutic target in the treatment of human melanoma.

\section{References}

1. Tas F: Metastatic behavior in melanoma: Timing, pattern, survival, and influencing factors. J Oncol 2012: 647684, 2012.

2. Geller AC, Clapp RW, Sober AJ, Gonsalves L, Mueller L, Christiansen CL, Shaikh W and Miller DR: Melanoma epidemic: An analysis of six decades of data from the Connecticut Tumor Registry. J Clin Oncol 31: 4172-4178, 2013.

3. Mehlen P and Puisieux A: Metastasis: A question of life or death. Nat Rev Cancer 6: 449-458, 2006.

4. Lamalice L, Le Boeuf F and Huot J: Endothelial cell migration during angiogenesis. Circ Res 100: 782-794, 2007.

5. Schaafhausen MK, Yang WJ, Centanin L, Wittbrodt J, Bosserhoff A, Fischer A, Schartl M and Meierjohann S: Tumor angiogenesis is caused by single melanoma cells in a manner dependent on reactive oxygen species and NF- $\kappa$ B. J Cell Sci 126 3862-3872, 2013.

6. Ellis LM and Hicklin DJ: VEGF-targeted therapy: Mechanisms of anti-tumour activity. Nat Rev Cancer 8: 579-591, 2008.

7. Welti J, Loges S, Dimmeler S and Carmeliet P: Recent molecular discoveries in angiogenesis and antiangiogenic therapies in cancer. J Clin Invest 123: 3190-3200, 2013.

8. Jia X, Hong Q, Lei L, Li D, Li J, Mo M, Wang Y, Shao Z, Shen Z, Cheng J, et al: Basal and therapy-driven hypoxia-inducible factor- $1 \alpha$ confers resistance to endocrine therapy in estrogen receptor-positive breast cancer. Oncotarget 6: 8648-8662, 2015.

9. Yeh JJ and Kim WY: Targeting tumor hypoxia with hypoxia-activated prodrugs. J Clin Oncol 33: 1505-1508, 2015.

10. Cao D, Zhou H, Zhao J, Jin L, Yu W, Yan H, Hu Y and Guo T: PGC-1 $\alpha$ integrates glucose metabolism and angiogenesis in multiple myeloma cells by regulating VEGF and GLUT-4. Oncol Rep 31: 1205-1210, 2014.

11. Zhang C, Li Y, Cornelia R, Swisher S and Kim H: Regulation of VEGF expression by HIF-1 $\alpha$ in the femoral head cartilage following ischemia osteonecrosis. Sci Rep 2: 650, 2012

12. Wei LH, Kuo ML, Chen CA, Chou CH, Lai KB, Lee CN and Hsieh CY: Interleukin-6 promotes cervical tumor growth by VEGF-dependent angiogenesis via a STAT3 pathway. Oncogene 22: 1517-1527, 2003.

13. Kimura H, Weisz A, Kurashima Y, Hashimoto K, Ogura T, D'Acquisto F, Addeo R, Makuuchi M and Esumi H: Hypoxia response element of the human vascular endothelial growth factor gene mediates transcriptional regulation by nitric oxide: Control of hypoxia-inducible factor-1 activity by nitric oxide. Blood 95: 189-197, 2000.

14. Levy DE and Darnell JE Jr: Stats: Transcriptional control and biological impact. Nat Rev Mol Cell Biol 3: 651-662, 2002.
15. Zhuang S: Regulation of STAT signaling by acetylation. Cell Signal 25: 1924-1931, 2013.

16. Mora LB, Buettner R, Seigne J, Diaz J, Ahmad N, Garcia R, Bowman T, Falcone R, Fairclough R, Cantor A, et al: Constitutive activation of Stat 3 in human prostate tumors and cell lines: Direct inhibition of Stat 3 signaling induces apoptosis of prostate cancer cells. Cancer Res 62: 6659-6666, 2002.

17. Niu G, Wright KL, Huang M, Song L, Haura E, Turkson J, Zhang S, Wang T, Sinibaldi D, Coppola D, et al: Constitutive Stat 3 activity up-regulates VEGF expression and tumor angiogenesis. Oncogene 21: 2000-2008, 2002.

18. Rao RA, Dhele N, Cheemadan S, Ketkar A, Jayandharan GR, Palakodeti D and Rampalli S: Ezh2 mediated H3K27me3 activity facilitates somatic transition during human pluripotent reprogramming. Sci Rep 5: 8229, 2015.

19. Caganova M, Carrisi C, Varano G, Mainoldi F, Zanardi F, Germain PL, George L, Alberghini F, Ferrarini L, Talukder AK, et al: Germinal center dysregulation by histone methyltransferase EZH2 promotes lymphomagenesis. J Clin Invest 123: 5009-5022, 2013.

20. Lee JM, Lee JS, Kim H, Kim K, Park H, Kim JY, Lee SH, Kim IS, Kim J, Lee M, et al: EZH2 generates a methyl degron that is recognized by the DCAF1/DDB1/CUL4 E3 ubiquitin ligase complex. Mol Cell 48: 572-586, 2012.

21. Gunawan M, Venkatesan N, Loh JT, Wong JF, Berger H, Neo WH, Li LY, La Win MK, Yau YH, Guo T, et al: The methyltransferase Ezh2 controls cell adhesion and migration through direct methylation of the extranuclear regulatory protein talin. Nat Immunol 16: 505-516, 2015.

22. Tong Q, He S, Xie F, Mochizuki K, Liu Y, Mochizuki I, Meng L, Sun $\mathrm{H}$, Zhang Y, Guo Y, et al: Ezh2 regulates transcriptional and posttranslational expression of T-bet and promotes Th1 cell responses mediating aplastic anemia in mice. J Immunol 192: 5012-5022, 2014

23. Kim E, Kim M, Woo DH, Shin Y, Shin J, Chang N, Oh YT, Kim H, Rheey J, Nakano I, et al: Phosphorylation of EZH2 activates STAT3 signaling via STAT3 methylation and promotes tumorigenicity of glioblastoma stem-like cells. Cancer Cell 23: 839-852, 2013.

24. Domashenko AD, Wiener S and Emerson SG: NF-Ya protein delivery as a tool for hematopoietic progenitor cell expansion. Methods Mol Biol 916: 303-316, 2012.

25. Lin YC, Chen YN, Lin KF, Wang FF, Chou TY and Chen MY: Association of 21 with NF-YA suppresses the expression of Polo-like kinase 1 and prevents mitotic death in response to DNA damage. Cell Death Dis 5: e987, 2014.

26. Domashenko AD, Danet-Desnoyers G, Aron A, Carroll MP and Emerson SG: TAT-mediated transduction of NF-Ya peptide induces the ex vivo proliferation and engraftment potential of human hematopoietic progenitor cells. Blood 116: 2676-2683, 2010.

27. Jin S, Fan F, Fan W, Zhao H, Tong T, Blanck P, Alomo I, Rajasekaran B and Zhan Q: Transcription factors Oct-1 and NF-YA regulate the p53-independent induction of the GADD45 following DNA damage. Oncogene 20: 2683-2690, 2001.

28. Matuoka K and Chen KY: Possible role of subunit A of nuclear factor Y (NF-YA) in normal human diploid fibroblasts during senescence. Biogerontology 1: 261-271, 2000.

29. Orgaz JL, Pandya P, Dalmeida R, Karagiannis P, SanchezLaorden B, Viros A, Albrengues J, Nestle FO, Ridley AJ, Gaggioli C, et al: Diverse matrix metalloproteinase functions regulate cancer amoeboid migration. Nat Commun 5: 4255, 2014.

30. Eustace AJ, Crown J, Clynes M and O'Donovan N: Preclinical evaluation of dasatinib, a potent Src kinase inhibitor, in melanoma cell lines. J Transl Med 6: 53, 2008.

31. Herraiz C, Calvo F, Pandya P, Cantelli G, Rodriguez-Hernandez I, Orgaz JL, Kang N, Chu T, Sahai E and Sanz-Moreno V: Reactivation of p53 by a cytoskeletal sensor to control the balance between DNA damage and tumor dissemination. J Natl Cancer Inst 108: pii: djv289, 2015.

32. Judd LM, Menheniott TR, Ling H, Jackson CB, Howlett M, Kalantzis A, Priebe W and Giraud AS: Inhibition of the JAK2/STAT3 pathway reduces gastric cancer growth in vitro and in vivo. PLoS One 9: e95993, 2014.

33. Zhou X, Ren Y, Liu A, Han L, Zhang K, Li S, Li P, Li P, Kang C, Wang X, et al: STAT3 inhibitor WP1066 attenuates miRNA-21 to suppress human oral squamous cell carcinoma growth in vitro and in vivo. Oncol Rep 31: 2173-2180, 2014. 
34. Garipov A, Li H, Bitler BG, Thapa RJ, Balachandran S and Zhang R: NF-YA underlies EZH2 upregulation and is essential for proliferation of human epithelial ovarian cancer cells. Mol Cancer Res 11: 360-369, 2013

35. Nishida N, Yano H, Nishida T, Kamura $\mathrm{T}$ and Kojiro $\mathrm{M}$ : Angiogenesis in cancer. Vasc Health Risk Manag 2: 213-219, 2006.

36. Xue Y, Lim S, Yang Y, Wang Z, Jensen LD, Hedlund EM Andersson P, Sasahara M, Larsson O, Galter D, et al: PDGF-BB modulates hematopoiesis and tumor angiogenesis by inducing erythropoietin production in stromal cells. Nat Med 18: 100-110, 2011.
37. Mahabeleshwar GH and Byzova TV: Angiogenesis in melanoma. Semin Oncol 34: 555-565, 2007.

38. Shibuya M: Vascular endothelial growth factor (VEGF) and its receptor (VEGFR) signaling in angiogenesis: A crucial target for anti- and pro-angiogenic therapies. Genes Cancer 2: 1097-1105, 2011.

39. Detmar M: Tumor angiogenesis. J Investig Dermatol Symp Proc 5: 20-23, 2000 